\title{
Validity and Reliability of the Persian Version of Conners' Early Childhood-Teacher/Childcare Provider ADHD Rating Scale
}

\author{
${ }^{1}$ Shahrokh Amiri, ${ }^{2}$ Ayyoub Malek, ${ }^{* 3}$ Homayoun Sadeghi-Bazargani, ${ }^{4}$ Amirhoushang Ghorashizadeh, \\ ${ }^{5}$ Salman Abdi, ${ }^{6}$ Masoumeh Ahsani, ${ }^{7}$ Ali Baharigharehgoz \\ ${ }^{1,2}$ Professor of Child and Adolescent Psychiatry, Research Center of Psychiatry and Behavioral Sciences, Tabriz \\ University of Medical Sciences, Tabriz, Iran. \\ ${ }^{* 3}$ Associate Professor of Epidemiology: Road Traffic Injury Research Center, Tabriz University of Medical \\ Sciences, Tabriz, IR Iran. \\ ${ }^{4,5}$ Resident of Psychiatry, Research Center of Psychiatry and Behavioral Sciences, Tabriz University of Medical \\ Sciences, Tabriz, Iran. \\ ${ }^{6}$ Psychologist, Road Traffic Injury Research Center, Tabriz University of Medical Sciences, Tabriz, Iran. \\ ${ }^{7}$ Student of Psychology \& Education of Exceptional Children, University of Allameh Tabataba'i, Tehran, Iran \\ Email:homayoun.sadeghi@gmail.com
}

\section{Received: $28^{\text {th }}$ February 2018, Accepted: $17^{\text {th }}$ March 2018, Published: $31^{\text {st }}$ October 2018}

\begin{abstract}
Objectives: to assess the validity and reliability of the Persian version of Conners' Early Childhood (EC)Teacher/Childcare Provider ADHD Rating Scale.

Methodology: Conners' EC-Teacher was translated by two native bilingualsfrom English to Persian and then back-translated into English. The Persian version was approvedby specialists in terms of consistency with the original version. A total of 1013 children of 3 to 6 years were randomly selected through the cluster sampling method from kindergartens and preschoolsin Tabriz and the two forms of Conners'EC (teacher and parents) were completed. The validity was determined through the Strengths and Difficulties Questionnaire (SDQ). To determine the diagnostic value, 334 children were interviewed using the clinical trials of DSM-IV TR criteria. The Receiver Operating Characteristic (ROC) curve was used to determine the best cutoff point.

Findings: The content validity for all contents was obtained using a modified Kappa of higher than 0.76 . The reliability of Conners' EC-Teacher with Cronbach's alpha coefficient was 0.91 and the retest reliability was $\mathrm{ICC}=0.89$. According to the convergence validity using $\mathrm{SDQ}$, the tool was accepted for evaluating the symptoms of ADHD. The ROC curve was evaluated with normal scores as well as the mean scores of parents and teacher forms. According to the findings, by eliminating $10 \%$ of people with a normal difference in the scores of teacher and parents, the area under the curve of the diagnostic value increased 0.77 and 0.79 , respectively.

Conclusion: The Persian version of Conners' EC-Teacher has a good validity and reliability. Simultaneous use of teacher and parentsforms and considering a minimum difference of $90 \%$ for teacher and parents scores hasa better diagnostic value.
\end{abstract}

Keywords

ADHD, Validity, Conners' EC-Teacher, Psychometrics, Persian

\section{Introduction}

According to the statistical and diagnostic manual, ADHD is one of the most common psychiatric diagnoses in children and adolescents (1). According to a recent report, the incidence of ADHD in Tabriz, Iran, is estimated at $9.8 \%$ in primary school children (2). Studies have shown that ADHD children are affected by the disease in different psychological aspectsand social relationships $(3,4)$, substance abuse $(5)$, and sleep problems $(6,7)$.On the other hand, ADHD is a heterogeneous disorder which is associated with several disorders (8). Given the damages in people with ADHD, an accurate and simple screening tool is a necessity for diagnostic interviews and performing therapeutic interventions.

The problems of screening tools for students are somewhat solved due to easier access to student children. But since ADHD starts before the age of 7, and ADHD is more prevalent in preschool age (9), therefore, developing a tool with accurate validity and trustworthy reliability is a challenge in psychometry of screening ADHD in 2 to 7 years old children. This is mainly due to the difficulty of sample collectionin this age range population (10, 11). Usually, a parent/guardian or teacherreport questionnaire is used to identify children suspected to ADHD. This per se complicates the diagnosis process and the measurement criteria, because convergence of parents and teacher forms is a controversial issue.

Using parent-report and teacher-report questionnaires, a study in Japan evaluated the prevalence of ADHD symptoms, according to which the prevalence of ADHD symptoms in boys and girls was reported as $36.2 \%$ and 
$25.7 \%$ (total mean $31.1 \%$ ), respectively, by parents and $7 \%$ and $1.5 \%$ (total mean $4.3 \%$ ), respectively, by teachers (12).

In another study in Ahwaz using the Conners' tool, the prevalence of ADHD in pre-school boys was reported $4.51 \%$ by parents and $17.29 \%$ by teachers. According to the reports of both teacher and parents, the prevalence was $25.2 \%$. Also using the scale, the total prevalence regardless of the person reporting, was estimated $24.06 \%$ (13).In another study, there was no relationship between parent-report and teacher-report regarding ADHD in preschool children (14).

Conners (2010) is one of the world's leading researchers in developing normalized tools for diagnosingADHD in children and adolescents. Conners has provided a toolfor screening ADHD at ages 2 to 7 years, which is reported in two forms of parents and teacher. Conners' Early Childhood Behavior Scale is a universal tool for screening ADHDfrom non-ADHD children. The scale consists of 115 items with 11 subscales in the parents form and 10 subscales in the teacher form.ADHD index is an important screening subscale (15). Considering the problemof time in screening ADHD children, inconsistencies in the parent-report and teacher-report forms, and the lack of an accurate tool in Iran were the main incentives for this study. Therefore, this study aimed to determine the validity and reliability of the Persian version of the Conners' Early Childhood ADHD Rating Scale in children 2 to 6 years and 11 months.

\section{Methodology}

This cross-sectional study was conducted in 2016. The statistical population consisted of all 3 to 6 years and 11 months old children in kindergartens and preschoolsin Tabriz northwest of Iran. There are a total of 10,000 children of 3 to 6 years and 11 months old in 160 kindergartens and preschools in Tabriz. The subjects were randomly selected through the cluster, consecutive sampling method from the kindergartens and preschoolsin Tabriz, regardless of gender. Given the research objectives and to have a larger sample size, a total of 1013 children were selected from 43 kindergartens and preschools from June 1, 2015 to June 1, 2016.In this study, diagnosing of ADHD was done based on clinical interview by two psychiatrist using DSM-IV criteria.

Inclusion and exclusion criteria

Consent of teacher and parents to participate in the study,reading and writing skills for parentsand minimally 2 tomaximally 6 years and 11 months old age of children were the inclusion criteria.Childrenunder psychiatry treatment due to mental disorder were excluded in the sampling stage.

Measurements

Conners' Early Childhood-Teacher

The Conners' Early Childhood Behavior Scale was developed by Conners (2010). The scale has 110 items with 3 impairment items and 2 additional items, making a total of 115 items. Like the parents version, Conners' ECTeacher has 10 subscales and a subset of additional sleep problems. Items are scored in a scalable scale in the form of a four-degree rating scale (from $0=$ never to $3=$ very high). Conners' internal reliability for the subscales of the scale is ranging from 0.76 to 0.94 according to Cronbach's alpha coefficient and the retest reliability is reported as 0.90 . Conners has confirmed the construct validity using factor analysis (15).

Conners' Early Childhood-Parent

The Conners' Early Childhood Behavior Scale was developed by Conners (2010). The scale has 110 items with 3 impairment items and 2 additional items, making a total of 115 items. Like the teacher version, Conners' ECParent has 10 subscales and a subset of additional sleep problems. Items are scored in a scalable scale in the form of a four-degree rating scale (from $0=$ never to $3=$ very high).

Conners has rated the evaluator's validity as 0.75 and the internal reliability by Cronbach's alpha coefficient for the subscales of this scale between 0.71 and 0.87 . The retest reliability was reported to be 0.88 .Conners has confirmed the construct validity using factor analysis (15).In Iran, Ahsani (16) reported the reliability using Cronbach's alpha coefficient as 0.72 and confirmed its diagnostic validity.

Strengths and Difficulties Questionnaire (SDQ)

SDQ has 25 items to measure children's problems in four areas (emotional symptoms, conduct problems, hyperactivity/inattention, peer problems)and an ability (prosocial behavior). SDQ is scored in the form of a three-degree rating scale $(0=$ not true, $1=$ sometimes true, $2=$ surely true) (17).In Iran, this checklist was applied on 3-17 years old subjects by Ghanizadeh et al.(18)and the reliability index of 0.62 is reported through the Cronbach's alpha coefficient. It is also approved in terms of sufficient convergent and discriminant validity. In this study, the ADHD subscale was used for investigating the concurrent validity of ADHD subscale of Conners' EC-Teacher tool.

Procedure

The scale distribution team collected the required data by referring to kindergartens and preschoolsand obtaining consent of parents and teachers. In order to measure the diagnostic value of sensitivity and specificity, a total of 700 children were randomly invited to conduct clinical interviews, of whom, only 334 children participated in clinical interviews. Also, the agreement level of the teacher version with the parent version was evaluated through using both Conners' EC versions. In this study, 35 scales were completed twice with a 2-week interval 
by teachers.All rules relating to ethical considerations and consent of parents and teachers were observed before performing the study.

The Conners' EC-Teacher Scale was as follows; the scale was first translated into Persian by a research group including a fully fluent English (native) speaker and a psychiatrist at the beginning of the study and in January 2016. The Persian version was examinedin terms of accuracy and was editedaccording to the views of English and Persian professors. At the same time, the necessary changes were applied by a panel of experts including 8 psychiatrists, 2 psychologists, and 1 methodologist considering formal and conceptual compliance, and the formal and content validity were determined.Then, the Persian version of the scale was translated back into English by a native English speaking person living in Iran without seeing the original English version. Theresearch team matched the concept of the translated sentences with the original ones (English), and finally, the Persian scale was finalized after confirmation.In this study, only the ADHD subscalewas studied in terms of validity and reliability.

\section{Statistical analysis}

All findings were analyzed by SPSS-21. Internal consistency was determined using the Cronbach's alpha coefficient and ICC. Test-retest reliability was calculated using the Pearson correlation coefficient. Content validity used in this study was the modified Kappa; this index is preferred to traditional I-CVI, because it also measures odds agreement. This index is represented by $\mathrm{K}^{*}$ and is calculated as follows $(\mathrm{A}=$ number of agreement on good fit, $\mathrm{N}=$ number of specialists):

$$
\begin{aligned}
& P c=\left[\frac{N !}{A !(N-A) !}\right] \cdot 5^{N} \\
& k^{*}=\frac{I-C V I-P c}{1-P c}
\end{aligned}
$$

In order to measure the diagnostic value of the scale, the ROC curve and area under the curve were used. $P$ value $<0.05$ was considered statistically significant in all statistical stages.

\section{Results}

A total of 1013 children aged 3 to 6 years and 11 months were participated in this study. The mean age of fathers was $34.13 \pm 4.60$ years with a maximum and minimum age of 24 and 65 years. The mean age of mothers was $39.37 \pm 5.71$ years with a minimum and maximum age of 23 and 50 years. The majority of parents had a high school diploma, a majority of fathers were employees and mothers were housewives (Table 1).

\begin{tabular}{|l|l|c|c|}
\hline Characteristics & Groups & N & \% \\
\hline \multirow{5}{*}{ Gender } & Girl & 569 & 56.2 \\
\cline { 2 - 4 } & Boy & 444 & 43.8 \\
\hline \multirow{5}{*}{ Father education } & Illiterate/Primary & 30 & 2.96 \\
\cline { 2 - 4 } & Middle & 138 & 13.62 \\
\cline { 2 - 4 } & Diploma & 303 & 29.91 \\
\cline { 2 - 4 } & Associate degree & 77 & 7.60 \\
\cline { 2 - 4 } & Bachelor & 286 & 28.23 \\
\cline { 2 - 4 } & Master & 179 & 17.67 \\
\hline \multirow{5}{*}{ Fother education } & Illiterate/Primary & 29 & 2.86 \\
\cline { 2 - 4 } & Middle & 82 & 8.09 \\
\cline { 2 - 4 } & Diploma & 366 & 36.13 \\
\cline { 2 - 4 } & Associate degree & 116 & 11.45 \\
\cline { 2 - 4 } & Bachelor & 336 & 33.16 \\
\cline { 2 - 4 } & Master & 84 & 8.29 \\
\hline & Unemployed & 19 & 1.9 \\
\cline { 2 - 4 } & Worker & 206 & 20.3 \\
\cline { 2 - 4 } & Employee & 417 & 41.1 \\
\cline { 2 - 4 } & Employer & 333 & 32.9 \\
\cline { 2 - 4 } & Military & 19 & 1.9 \\
\cline { 2 - 4 } & Retired & 19 & 1.9 \\
\hline \multirow{5}{*}{ Mother occupation } & Housewife & 162 & 168.3 \\
\cline { 2 - 4 } & Government employee & 159 \\
\cline { 2 - 4 } & Private sector employee & 16.7 \\
\hline
\end{tabular}

Table 1: Chi-Square Test Results of Education, Employment, and Gender 
Reliability

Using Cronbach's alpha, the internal reliability of the Persian version of the Conners' Early Childhood-Teacher ADHD Rating Scale was calculated as 0.91 . The retest reliability was $\mathrm{ICC}=0.89$ and the Pearson correlation coefficient was 0.82 .

Validity

Using the modified Kappa, the content validity of the Persian version of the Conners' Early Childhood-Teacher was obtained above 0.76 in 10 specialist samplesfor all items. Using Pearson correlation coefficient, the concurrent validity of the Conners' Early Childhood-Parent ADHD Rating Scale was calculated as 0.26, while using SDQ, theconcurrentvalidity of the ADHD subscale was calculated as 0.73 .

Evaluation of diagnostic value

To evaluate the diagnostic value of Conners' EC-Teacher, 334 children were assessed for ADHD through the SADS-K-PL semi-structured diagnostic interview according to DSM-IV criteria; of these, 129 (38.62\%) were diagnosed with ADHD. After analyzing the results of clinical interviews, the diagnostic value of the Persian version was analyzed by the ROC curve.

In assessing the mean raw scores of the scale, the total score of items was calculated and then the subjects' scores were calculated in the normal score (a) from zero to 100 . The relative difference index (d) is deduced from the difference between the normal score of teacher and the normal score of parents divided by the mean raw score of each person.

$$
\begin{aligned}
& a=\text { average }-N P T=\frac{N \text { teacher } A D H D+N \text { parent } A D H D}{2} \\
& d=\text { deff-relation }=\frac{\text { deff } N P T}{\text { average }} \times 100
\end{aligned}
$$

The results of the ROC curve showed that with a normal teacher scores of $68 \%$ and average values of normal NPT, $73 \%$ of the area under the curve is covered with a $95 \%$ confidence interval (CI). Evaluation of cutoff pointsshowed that in the best cutoff point, the teacher normal score is 14.16 , sensitivity is $69 \%$, and specificity is $57 \%$.In addition, the average scores of teacher and parentsshowed the best cutoff point of 20.41 with a sensitivity of $73 \%$ and a specificity of $62 \%$. Therefore, it seems that by interfering the normal scores of both scales (Conners' Teacher and Parents Forms), the amounts of diagnostic value are relatively improved (Table 2). By using the index of relative difference, the data of $10 \%$ of the participants with the highest difference between the scores obtained fromteachersand parents were excluded and 937 peoplewere selected.The ROC curve showed that the area under the ROC curve is $77 \%$ with the normal data of teacher and the average normal scores of parent-teacher is $79 \%$, which is in $95 \%$ confidence interval (CI). Evaluation of cutoff points revealed that at the best cutoff point, the teacher normal score is 22.50 with a sensitivity of $73 \%$ and a specificity of $60 \%$. Also, the average scores of teacher and parents at the best cutoff point is 22.08 with a sensitivity of $81 \%$ and a specificity of $63 \%$ (Table 3 ).

\begin{tabular}{cccccc}
\hline \multicolumn{1}{c}{ Normal Score } & & \multicolumn{3}{c}{ Average TP } \\
\hline 9.16 & Sensitivity & Specificity & Score & Sensitivity & Specificity \\
10.83 & 0.84 & 0.30 & 14.68 & 0.86 & 0.38 \\
12.50 & 0.80 & 0.42 & 15.93 & 0.85 & 0.47 \\
14.16 & 0.74 & 0.49 & 17.08 & 0.82 & 0.52 \\
15.83 & 0.69 & 0.57 & 17.50 & 0.78 & 0.55 \\
17.50 & 0.66 & 0.58 & 18.54 & 0.77 & 0.56 \\
19.16 & 0.61 & 0.62 & 19.79 & 0.76 & 0.59 \\
20.83 & 0.60 & 0.63 & 20.41 & 0.73 & 0.62 \\
22.50 & 0.54 & 0.69 & 21.04 & 0.70 & 0.64 \\
\hline
\end{tabular}

Table 2: Sensitivity and Specificity of Conners' EC-Teacher for the Main Scores

\begin{tabular}{cccccc}
\multicolumn{3}{c}{ Table 2: Sensitivity and Specificity of Conners' EC-Teacher for the Main Scores } \\
\hline Normal Score & \multicolumn{3}{c}{ Average TP } \\
\hline 14.16 & Sensitivity & Specificity & Score & Sensitivity & Specificity \\
15.83 & 0.89 & 0.43 & 20 & 0.87 & 0.56 \\
17.50 & 0.85 & 0.44 & 20.72 & 0.85 & 0.58 \\
19.16 & 0.79 & 0.50 & 21.35 & 0.83 & 0.60 \\
20.83 & 0.77 & 0.52 & 22.08 & 0.81 & 0.64 \\
22.50 & 0.75 & 0.58 & 23.22 & 0.79 & 0.65 \\
24.16 & 0.72 & 0.60 & 24.06 & 0.75 & 0.65 \\
25.83 & 0.70 & 0.66 & 24.58 & 0.72 & 0.67 \\
27.50 & 0.66 & 0.72 & 25.72 & 0.70 & 0.69 \\
& 0.58 & 0.78 & 27.39 & 0.68 & 0.71 \\
\hline
\end{tabular}




\section{Table 3: Sensitivity and Specificity of Conners, EC-Teacher for less than $90 \%$ of Difference in Relative Scores of Teachers and Parents}

\section{Discussion}

This is the first study on the psychometric properties of the Persian version of the Conners' EC-Teacher ADHD Rating Scale in Iran. The results showed that using modified CVI (modified kappa), the content validity in 10 specialist samples was above 0.76 for all items. Therefore, the Persian version is of good content validity with no need to change.

The reliability of Cronbach's alpha coefficient was 0.91 and the retest reliability was $\mathrm{ICC}=0.89$. This finding implies the very good results of internal reliability. Conners (15) also pointed out 0.82 and 0.90 for ADHD scale.

The findings showed that the concurrent validity of the two ADHD scales of the Conners' EC-Teacher tool and SDQ was 0.73.Conners (15) has also pointed out a high convergence validity between Conners' EC-Teacher and Teacher Rating Scales-Preschool (TRS-P) in the attention subscale of 0.85 and the hyperactivity subscale of 0.90.This finding implies the agreement between ADHD symptoms and the symptoms reported in other questionnaires for ADHD assessment.

Using the Pearson correlation coefficient, the concurrent validity of the ADHD subscale of Conners' Parents and Teacher Forms was obtained 0.26. However, Conners (15) has reported a correlation coefficient of 0.88 between the two versions of parents and teacher for the ADHD subscale. This finding has a big difference with Conners' results. It should be noted that previous studies have also suggested differences in the agreement of teacher and parents in the symptoms of ADHD children $(19,20)$.

In this regard, a similar study in Japan evaluated the prevalence of ADHD symptoms, according to which the prevalence of ADHD symptoms in boys and girls was reported as $36.2 \%$ and $25.7 \%$ (total mean $31.1 \%$ ), respectively, by parents and $7 \%$ and $1.5 \%$ (total mean $4.3 \%$ ), respectively, by teachers (21).

The results of the ROC curve showed that with a normal teacher scores of $68 \%$ and average values of normal NPT, $73 \%$ of the area under the curve is covered with a $95 \%$ confidence interval (CI). The best cutoff point of the teacher normal score is 14.16 , sensitivity is $69 \%$, and specificity is $57 \%$. In addition, the average scores of teacher and parents showed the best cutoff point of 20.41 with a sensitivity of $73 \%$ and a specificity of $62 \%$. Therefore, it seems that by interfering the normal scores of both scales (Conners' Teacher and Parents Forms), the amounts of diagnostic value are relatively improved. According to the findings of Conners in terms of diagnostic value, the reference values for sensitivity and specificity of the main tool was $89 \%$ and $91 \%$, respectively. This finding is different from the results of Conners (15).It seems that considering both the parents and teacher indices for screening can reduce the geographical differences in the sensitivity and specificity of the Conners' EC-Teacher tool.

Since the diagnosis of parents and teachers is important, in the second step of this study, $90 \%$ of the data of children's parents and teachers with the least difference in ADHD symptoms were selected and analyzed. The results showed that the mean scores of teacher and parents at the best cutoff point is 22.08 with a sensitivity of $81 \%$ and a specificity of $63 \%$.Although cultural and personality differences of teachers and parents, as well as the answering culture can be a main reason for the difference in results, the geographical differences in the use of the tool can be the most important factor in this regard.

Undoubtedly, the use of a research tool that has sufficient validity and reliability can improve screening accuracy and help its widespread use. Therefore, the present study confirms the usefulness of Conners' ECTeacher for screening and emphasizes the importance of simultaneous use of both parents and teachers. It is important to note that the use of the results of every study will be useful in the context of its constraints. Therefore, it is important to consider the geographical location of the study, use both parents and teacher forms, andtake into account that the difference of more than $90 \%$ cannot demonstrate the correctness of symptoms by parents and teachers.

\section{Conclusion}

Considering the results of studies conducted in different countries, it seems that Conners' EC-Teacher should be localized and a cutoff should be determined for every community.However, the findings of this study showed that the Persian version of Conners' EC-Teacher is of good validity for the diagnosis of ADHD. Since identifying children with ADHD in early life is important for treatment interventions, Conners' EC-Teacher can be used for initial screening. But it should be considered that the diagnosis of ADHD based only on the results of the existing questionnaires is not recommended; instead, this tool can only be used as a helping tool in conjunction with a clinical interview for screening.

\section{Conflict of Interest}

The findings of this study have no relationto the interests of the authors. 


\section{Acknowledgment}

All parents and teachers involved in this study are appreciated.

\section{References}

1- American Psychiatric Association. Diagnostic and statistical manual of mental disorders. Text revision. 4th ed. Washington D.C: American psychiatric association press; 2000.

2- Amiri S, Fakhari A, Maheri M, Mohammadpoor Asl A. Attention deficit/hyperactivity disorder in primary school children of Tabriz, North-West Iran. Paediatric and Perinatal Epidemiology 2010; 24: 597-601.

3- de Boo GM, Prins PJ. Social incompetence in children with ADHD: possible moderators and mediators in social-skillstraining, ClinPsychol Rev. 2007 Jan;27(1):78-97.

4- Olaniyan O, dosReis S, Garriett V, Mychailyszyn MP, Anixt J, Rowe PC, Cheng TL Community perspectives of childhood behavioral problems and ADHD among African American parents, AmbulPediatr.2007;7(3):226-31.

5- Lee SS, Humphreys KL, Flory K, Liu R, Glass K. Prospective association of childhood attentiondeficit/hyperactivity disorder (ADHD) and substance use and abuse/dependence: a meta-analytic review, ClinPsychol Rev 2011;31(3):328-41.

6- Sciberras E, Fulton M, Efron D, Oberklaid F, Hiscock H.Managing sleep problems in school aged children with ADHD: a pilotrandomised controlled trial, Sleep Med. 2011 Oct;12(9):932-5.

7- Jan YW, Yang CM, Huang YS.Comorbidity and confounding factors in attention-deficit/hyperactivity disorder and sleep disorders in children, Psychol Res BehavManag 2011;4:139-50.

8- Gargaro BA, Rinehart NJ, Bradshaw JL, Tonge BJ, Sheppard DM. Autism and ADHD: how far have we come in the comorbidity debate? Neurosci Biobehav Rev. 2011 Apr;35(5):1081-8.

9- Soma Y, Nakamura K, Oyama M, Tsuchiya Y, Yamamoto M. Prevalence of attention-deficit/hyperactivity disorder (ADHD)symptoms in preschool children: discrepancy between parent and teacher evaluations, Environ Health Prev Med 2009; 14:150-154.

10- HebraniP, Abdolahian E, Behdani F, Vosoogh I, Javanbakht A. The Prevalence of Attention Deficit Hyperactivity Disorder in Preschool-Age Children in Mashhad, North-East of Iran, Arch Iranian Med 2007; $10(2): 147-151$.

11- Wilens TE, Biederman J, Brown S, Tanguay S,Monuteaux MC, Blake C, et al. Psychiatric comorbidity and functioning in clinically referred preschool children and school-age youths with ADHD. J Am Acad Child Adolesc Psychiatry. 2002; 41: $262-268$

12- Soma Y, Nakamura K, Oyama M, Tsuchiya Y, Yamamoto M. Prevalence of attention-deficit/hyperactivity disorder (ADHD) symptoms in preschool children: discrepancy between parent and teacher evaluations, Environ Health Prev Med 2009; 14:150-154.

13- TashakoriA, Afkandeh R. Prevalence of ADHD Symptoms among eMale Preschoolers Basedon Different Informants in Ahvas City of Iran, ISRN Pediatrics 2011, Volume 2011, Article ID 709653, 4 pages.

14- SimsDM, Lonigan CJ. Multi-method assessment of ADHD characteristics in preschool children :Relations between measures, Early Childhood Research Quarterly 2011, Article in Press.

15- Conners C. Conners Early Childhood, $3^{\text {rd }}$ Edition Conners 3. Canada: Torento, Multhi-Health System Inc, 2010.

16- Ahsani M. The evaluation of psychometric properties of Persian Version of Conners Early ChildhoodTMParentin3 to 6 and 11 months aged children of Tabriz. Tabriz University of Medical Sciences. 2017.

17- Goodman R. Psychometric Properties of the Strengths and Difficulties Questionnaire. Journal of the American Academy of Child \& Adolescent Psychiatry. 2011; 40(11): 1337-1345.

18- Ghanizadeh A, Izadpanah A, Abdollahi G. Scale Validation of the Strengths and Difficulties Questionnaire in Iranian Children. Iranian Journal of Psychiatry 2007. 2(2):65-71.

19- Narad M, Garner A, Peugh J, Tamm L, Antonini T, Kingery M, Simon J, and Epstein J. Parent- Teacher Agreement on ADHD Symptoms Across Development. Psychol Assess 2015, 27(1): 239-248.

20- Sherman EM, Brooks BL, Akdag S, Connolly MB, WiebeS.Parents report more ADHD symptoms than do teachers in children with epilepsy. Epilepsy Behav. 2010 Nov;19(3):428-35. doi: 10.1016/j.yebeh.2010.08.015.

21- Soma Y, Nakamura K, Oyama M, Tsuchiya Y, Yamamoto M. Prevalence of attention-deficit/hyperactivity disorder (ADHD)symptoms in preschool children: discrepancy between parent and teacher evaluations, Environ Health Prev Med 2009; 14:150-154. 